\title{
La orientación de la política social en el ciclo de recuperación "posneoliberal" en Uruguay (2005-2015)*
}

\author{
The guidelines of the social policy in the "cycle \\ of post-iliberal recovery" in Uruguay (2005-2015)
}

Laura Vecinday

Doctora en Ciencias Sociales (Flacso), Master en Servicio Social (UFRJ-Universidad de la República (UdelaR), Asistente Social (UdelaR); Docente del Depto. de Trabajo Social (UdelaR), Montevideo, Uruguay.

lauravecinday@gmail.com

Resumen: El texto analiza la orientación general de la política social en el denominado "ciclo de recuperación posneoliberal", centrándose en el análisis de la experiencia uruguaya. Luego de delinear genéricamente y sin pretensiones de exhaustividad los principales rasgos de la política social en campos particulares, se analizan los efectos más de las reformas iniciadas en el período, advirtiendo sobre el carácter provisorio de cualquier afirmación acerca de procesos aún en curso.

Palabras clave: Política Social. Neoliberalismo. Uruguay.

\begin{abstract}
This article analyzes the general guidelines of the social policies in the so called "cycle of post-liberal recovery", centering in the analysis of the Uruguayan experience. After a general and non-comprehensive description of the main features of the social policy in particular fields, more effects of the reforms started in that period were analyzed, but the temporary character of any information about the processes still in progress must be taken into consideration.
\end{abstract}

Keywords: Social policy. Neoliberalism. Uruguay.

* Este trabajo fue presentado en el III Seminario Latinoamericano Desigualdad y políticas socio-laborales a propuesta del Grupo de Estudio de Políticas Sociales y Condiciones de Trabajo (GEPSyCT) del Instituto Gino Germani (FCS-UBA) realizado en mayo de 2015. 


\section{Antecedentes}

ara comprender la orientación general de la política social en el Uruguay
actual es innevitable trazar algunas líneas sobre la arquitectura de la
protección social sobre la cual se montan las alteraciones más recientes. Dos elementos son claves para interpretar el sentido y alcance de las rupturas y continuidades registradas en el período estudiado: i) la incipiente construcción del Estado Social uruguayo y ii) la introducción moderada y heterodoxa de las propuestas de reforma neoliberales (Moreira, 2001).

En las tres primeras décadas del siglo XX, el Estado uruguayo promovió una serie de iniciativas y regulaciones en materia social y laboral que permitieron caracterizarlo como "un Estado expandido que 'coloniza' prácticamente todas las dimensiones del espacio social” (Ortega, 2003, p. 24). Emerge tempranamente una matriz de seguridad social abarcativa "capaz de atender los problemas vinculados a la protección laboral, la transformación económica y la integración social, generando así las bases de una sociedad hiperintegrada e igualitaria" (Midaglia, 2000, p. 26). Este proceso de ampliación del Estado fue acompañado del pasaje de la visión de "privilegios" a la de "derechos sociales" (Castellanos apud Ortega, 2003, p. 35). La redistribución de la riqueza y la ampliación democrática se articularon elevando los niveles de consumo y bienestar de la población, lo que permitió a Real de Azúa acuñar la expresión "sociedad amortiguadora", en la medida en que se consolidaba una sociedad relativamente igualitaria que moderaba los potenciales conflictos sociales y políticos (Serna, 2005).

Sobre aquella estructura de bienestar intentaron incidir las propuestas neoliberales a partir de los 80 , impulsadas por los organismos internacionales en alianza con la "alta nobleza estatal" ", con el objetivo de reemplazar "las políticas intervencionistas y orientadas al mercado interno, incorporando las de apertura económica y mercado abierto y promoviendo un contexto intelectual favorable al neoliberalismo" (Orlansky, 2005, p. 8). Señala Moreira (2001) que los diferentes procesos de reforma en América Latina se explican por variables

1. "En el período contemporáneo, el campo burocrático se ve atravesado por dos luchas intestinas. La primera opone la 'nobleza estatal más alta' - la de los políticos que promueven reformas orientadas al mercado - y la 'nobleza estatal más baja', la de los ejecutores ligados a las misiones tradicionales del gobierno. La segunda opone lo que Bourdieu llama la 'mano izquierda' y la 'mano derecha' del Estado" (Wacquant, 2011). 
de tipo político que determinaron la agenda, el procesamiento político y los mecanismos de institucionalización de las reformas. Agrega que, en el caso uruguayo, este proceso adoptó un carácter gradualista: "Uruguay implementó un conjunto de reformas en sus políticas públicas y en los instrumentos de regulación de los mercados que, tanto por su forma (gradualista) como por sus contenidos eclécticos y en casos claramente alejados del consenso de Washington, pueden ser considerados como únicos"(IDH, Uruguay, 1999 apud Moreira, 2001). Las luchas promovidas por la izquierda política, el movimiento obrero y otros actores sociales en oposición a las reformas impulsadas es una variable política sustantiva para comprender el gradualismo de las reformas.

En 2005 asume su primer gobierno nacional el Frente Amplio, principal fuerza política identificada con la izquierda. Siendo portador, en materia social, de una histórica oposición a los lineamientos del Consenso de Washington, delinea los trazos principales de lo que entiende es una "nueva concepción de la política social" contrapuesta a aquella dominante en los 1990. La "nueva concepción" afirma la responsabilidad del Estado como rector de la política social. Señala la necesidad de contar con un proyecto de desarrollo social que articule las políticas sociales con las económicas. Asimismo, subraya que la superación de la descoordinación y fragmentación institucional de las políticas sociales será consecuencia de la formulación de una estrategia de desarrollo social pensada desde los problemas a atender y no desde las instituciones. Se defiende el recurso a la focalización de las prestaciones, siempre que se articulen con políticas universales, dejen capacidades instaladas en los organismos rectores de la política social y se articulen territorialmente ${ }^{2}$.

\section{Esbozo de los principales trazos y rasgos de la política social (2005-2014)}

El gasto público social en el período confirma su tendencia histórica, que se ha mantenido en aumento durante todo el siglo XX, a pesar de bajas episódicas, por ejemplo, la registrada durante la crisis del 2002:

2. Comité de Coordinación Estratégica. Estrategia Nacional para la Infancia y la Adolescencia (2006-2010), nov. 2005. 
En Uruguay, el Gasto Público Social ha evolucionado desde un quinto hasta dos tercios del total del gasto público durante el siglo XX (...). El sostenido crecimiento del GPS en términos de su participación en el gasto público y en la riqueza es una característica de las finanzas públicas occidentales en el siglo XX. Uruguay no ha sido una excepción (...). Incluso en la década del 90, popularizada como la década del neoliberalismo, el Gasto Público Social sufrió un significativo aumento (Bentura; Mariatti, 2014, p. 78).

En el período, el gasto público social crece acompañado por el crecimiento de la economía en un "ciclo que ya lleva doce años de expansión ininterrumpida" (Instituto Cuesta Duarte, 2015).

En materia laboral, se reinstalan los reinstalados Consejos de Salarios como modalidad de negociación colectiva. Aumenta el salario mínimo y el conjunto de remuneraciones ("el salario real (...) creció por décimo año consecutivo"), se reduce la informalidad laboral ("los ocupados no cubiertos por la seguridad social se ubicó en $24,9 \%$ ") y los niveles de desempleo han alcanzado mínimos históricos (se "alcanzaron niveles de empleo y desempleo muy positivos" con una tasa de desocupación situada en 6,6\% en promedio) (Instituto Cuesta Duarte, 2015).

Para el Instituto Cuesta Duarte, la mayor participación de la masa salarial sobre el producto interno es el principal desafío pues

A pesar del histórico crecimiento salarial logrado por los trabajadores en la última década, la participación de la masa salarial total sobre el producto interno se encuentra en niveles inferiores a los guarismos del período previo al estallido de la última crisis, en 1998 (...). Además, un número importante de trabajadores siguen percibiendo ingresos que podríamos calificar como bajos o muy bajos (Instituto Cuesta Duarte, 2015).

La desigualdad de ingresos se encuentra prácticamente estancada: en 2014 el 20\% de hogares más ricos se apropió del 42,9\% del total de ingresos del país mientras que al 20\% más pobre le correspondió el 6,9\% del total de ingresos (Instituto Cuesta Duarte, 2015). Estos datos dan cuenta de que es posible reducir la pobreza y la indigencia y, al mismo tiempo, aumentar la desigualdad. Por 
lo tanto, aquí hay otro desafío que nos reenvía a los problemas de creciente concentración de la riqueza.

Si bien aumenta la capacidad organizativa de la clase trabajadora recuperando niveles de afiliación sindical como uno de los efectos de la reinstalación de los Consejos de Salarios, ello no trajo mayores niveles de conflictividad. Quizás la histórica relación de los sindicatos con los principales partidos que conforman el Frente Amplio y, una de sus expresiones, la participación de dirigentes sindicales como funcionarios del gobierno contribuyan en la comprensión de esta coyuntura.

En materia social, se diseña el Plan de Equidad con "componentes estructurales" que incluyen: la reforma tributaria, la reforma de la salud, la revisión de las políticas de empleo, una nueva política de vivienda y hábitat, la reforma educativa y el plan nacional de igualdad de oportunidades y derechos. Además, el Plan incluye un conjunto de "componentes específicos" que conforman una "Red moderna de Asistencia e Integración Social" (RAIS) estructurada en una serie de estrategias: transferencias monetarias no contributivas, medidas de seguridad alimentaria, políticas de educación para la infancia y adolescencia, políticas asociadas al trabajo protegido, promoción de cooperativas sociales y emprendimientos productivos, políticas de promoción e inclusión social y políticas de atención de la discapacidad ${ }^{3}$.

A modo de ordenar el análisis, se presenta una breve reseña de los procesos de innovación o reforma en los principales componentes del Plan de Equidad.

En materia tributaria, se construye un nuevo sistema que grava la renta de las personas físicas (IRPF), al tiempo que se realizan esfuerzos para reducir la carga de los impuestos indirectos. Como crítica principal sobre la orientación de esta reforma, se puede señalar que ha generado una redistribución dentro de los asalariados, fundamentalmente, afectando de forma residual a las rentas del capital.

Se implementan programas de trabajo protegido, capacitación laboral, promoción de cooperativas sociales y emprendimientos productivos, y regulaciones

3. Plan de Equidad. Disponible en: http://www.mides.gub.uy/innovaportal/file/913/1/plan_equidad_def. pdf. Acceso en: 20 ago. 2009. 
específicas dirigidos a los "inempleables" e "invalidados por la coyuntura" (Castel, 1997).

Sobre el acceso a los servicios de salud, se creó el Sistema Nacional Integrado de Salud (SNIS) que, a través del Fondo Nacional de Salud (FONASA) habilita a los trabajadores formales, jubilados, pensionistas y sus cónyuges e hijos a seleccionar su prestador de salud (público o privado) descontando un porcentaje de sus ingresos. La Administración de los Servicios de Salud del Estado (ASSE) es el prestador público y las instituciones de asistencia médica colectiva (IAMC) conforman el universo de prestadores privados junto a los seguros médicos. Se instala un sistema de competencias entre ambos para capturar a los "socios FONASA". El resultado provisorio de esta competencia es que, de hecho y tendencialmente, ASSE se encarga de la población alejada o precariamente integrada al mercado de trabajo y de los trabajadores formales cuyos ingresos no les permiten costear los copagos del sistema privado. La reforma en la atención sanitaria,

(...) ha tenido resultados positivos en términos de incorporación de grandes porciones de la población a la cobertura formal, entre otros factores. Pero al mismo tiempo, lejos de diluir las grandes diferencias heredadas entre los prestadores públicos y privados, las mismas - atenuadas - se han consolidado cada vez más, confirmando la estructura dual de provisión (Antía et al., 2013, p. 18).

Educación y vivienda han sido los grandes pendientes de los dos últimos gobiernos. En ambas áreas de política se registran dificultades importantes. Este tercer gobierno ha tomado el tema educativo como uno de los pilares a ser abordado en el período. Hubo tímidos anuncios en el momento electoral que rápidamente despertaron voces discrepantes desde dentro y fuera de la fuerza política gobernante y desde los sindicatos de la educación. Quizá esas primeras señales de resistencia motivaron el silencio que sobre el tema reina hoy en día.

En materia de acceso a la vivienda de interés social, se ensayaron algunas propuestas y se reformó la institucionalidad tradicional en el área pero hay acuerdo sobre los escasos avances y resultados obtenidos en el acceso a soluciones habitacionales. 
Respecto a la gestión del Estado en el campo social, se observan (i) innovaciones institucionales, con la emergencia del Ministerio de Desarrollo Social (MIDES) encargado de llevar adelante iniciativas dirigidas a los individuos pobres y/o minusválidos sociales en el sentido que le atribuye Castel (2003) y (ii) la adopción de medidas emparentadas con el paradigma de la "Nueva Gestión Pública (NGP)" (Narbondo, 2011).

Cómo interpretar estos procesos de innovación institucional es parte de los debates que atraviesan el período que intentamos analizar. La creación del MIDES puede ser un indicador del fracaso de la libertad absoluta del mercado y un retorno de la intervención social del Estado como respuesta ante los efectos sociales regresivos de la etapa anterior. Sin embargo, si nos detenemos a analizar la significación sociopolítica de esta creación institucional y su asignación presupuestal podríamos concluir que, efectivamente, hay un mínimo retorno del Estado o, como señala Midaglia (2012, s/d.), un leve "retorno del Estado".

Sin lugar a dudas, el MIDES expresa una visión muy limitada del desarrollo social reducido a los problemas de la pobreza y extrema pobreza mereciendo, más bien, denominarse Ministerio de Focalización Social, como señala Bentura (2014). Continuando esa línea, la reorientación de la gestión del MIDES promovida en este segundo período de gobierno se propuso para ofrecer "una mirada integral y concentrada en núcleos duros de pobreza" (Mides, 2015, p. 5). La creación de Ministerios Sociales puede interpretarse como expresión institucional de la disociación de la cuestión de la pobreza, por un lado, y la cuestión del trabajo, por otro. En este sentido, Midaglia (et al.) indica que la presencia de esta nueva institucionalidad

(...) pone de manifiesto que las problemáticas relacionadas con la pobreza y vulnerabilidad social tienden a perdurar, y en algún sentido, están "separadas", o mejor dicho, carecen de soluciones asociadas al mercado de empleo. Los Planes y políticas sociales que manejan estas Secretarías dejan en evidencia que se requiere de medidas específicas para tratar las actuales cuestiones sociales, y al parecer, esas iniciativas son tan particulares que se torna imposible o inconveniente ubicarlas en la esfera de los Ministerios de Trabajo (Midaglia et al., 2010, p. 150). 
La asistencia social — o la "seguridad social" no contributiva de la pobreza - es el campo de competencia institucional de los nuevos Ministerios Sociales (Vecinday, 2013).

Sobre el paradigma de la NGP se podría decir, si creyéramos en la neutralidad política que el modelo se autoatribuye, que se trata de transformaciones en la gestión del Estado que pueden responder tanto a orientaciones neoliberales como a orientaciones de signo político contrario (Narbondo, 2011). Narbondo (2011) nos recuerda que este paradigma surge con la crisis del Estado keynesiano de Bienestar y la expansión de las reformas neoliberales cuyo blanco ha sido la burocracia weberiana y que es contradictorio con una acción pública de orientación keynesiana. En esta línea de transformaciones de la gestión pública, se están implementando sistemas de información y vigilancia dirigidos a fortalecer la administración gerencial del Estado. Entre otros cometidos, estos sistemas han perfeccionado la función recaudadora del Estado minimizando la evasión fiscal.

A los efectos de este análisis, me interesa resaltar un objetivo en particular de la tecnificación de las funciones del Estado asociado a la necesidad de identificar y controlar a los beneficiarios de la asistencia. La creciente tecnificación del campo socio-asistencial persigue como objetivos la adecuada focalización, el seguimiento de las trayectorias de los individuos y familias atendidos, la verificación del cumplimiento de las condicionalidades, el control del trabajo profesional empleado y la identificación de recursos y coberturas superpuestos. Si bien la historia de la asistencia puede ser leída como la historia de su tecnificación (Castel, 1997), nos toca ser testigos de una época que, conservando en su espíritu la "ensoñación tecnocrática" (Castel, 1986) de ejercer un control y una prevención perfectos sobre las poblaciones y sobre los individuos, cuenta con las posibilidades instrumentales de aproximarse a su realización que, por cierto y por suerte, siempre será inacabada. No se trata de rechazar el uso de las nuevas tecnologías sino de problematizar sus usos sociales.

Por otro lado, gran parte de las reformas institucionales y tecnológicas propuestas para el campo social privilegian la "creación de nuevos dispositivos de trabajo social y de política social” (Merklen, 2013, p.8). La crítica a las instituciones de protección universal es el fundamento para la creación de nuevos instrumentos y de una nueva generación de programas para atender el 
"núcleo duro de la pobreza" con muy fuerte focalización, de carácter interinstitucional, con trabajo de proximidad y cercanía, anclados en el territorio y centrados en las trayectorias individuales y familiares (Mides, 2015). Estos programas, "by-pasean" a la institucionalidad típica de orientación universal y, paradójicamente, se plantean ser un mojón en el camino hacia la integración a estas prestaciones. Parece no advertirse que, al pretender operar desde fuera de la institucionalidad tradicional, el propio diseño institucional atenta contra esta estrategia y refuerza el riesgo de tránsitos paralelos ${ }^{5}$

\section{Síntesis de los principales efectos de la política social en el período}

Los principales efectos - no todos ellos esperados — de la orientación actual de las políticas sociales se pueden agrupar del siguiente modo:

1. Refuerzo de la consolidación de una matriz de protección dual dirigida a distintos segmentos de la clase trabajadora con prestaciones diferenciadas según la arena de política. Se diseñan esferas específicas y paralelas que "institucionalizan" trayectorias de protección diferenciadas para la clase trabajadora. Por ejemplo, el Ministerio de Trabajo se encarga de los trabajadores y la cuestión del trabajo mientras que las prestaciones del MIDES se dirigen a los segmentos más empobrecidos de la clase trabajadora (precarios, desocupados de larga duración etc.). En la atención sanitaria, ASSE, como prestador público, absorve el cuidado de los trabajadores desempleados, precarios o con ingresos miserables mientras que el sistema privado se orienta hacia aquellos cuyos ingresos les permiten cubrir los costos de esta atención.

4. La expresión es tomada de Midaglia (1998, p. 94) quien la utilizara para ilustrar el mecanismo a través del cual el PRIS - programa de inversión típico de los 90 — sorteaba los controles político-burocráticos y se situaba por fuera de la institucionalidad pública tradicional construyendo "vías paralelas o semi-paralelas no contaminadas por los 'vicios' clásicos de las intervenciones públicas".

5. El programa Cercanías es un ejemplo de ello. Se trata de un programa que surge como respuesta a la situación del 15\% más pobre y, en particular, del 5\% de pobreza estructural. Uno de sus objetivos es "la implementación de cambios en la gestión institucional de las políticas públicas para dar respuesta rápida e integral (...) a situaciones de vulnerabilidad, extrema pobreza e indigencia”. Extraído del documento "Estrategia Nacional de Fortalecimiento de las Capacidades Familiares. Modelo de Atención”. Programa Cercanías, diciembre de 2013. 
2. Leve aumento de la formalización del trabajo, disminución del desempleo y mejoras salariales. Respecto al comportamiento del mercado de trabajo, preocupa que el crecimiento de la ocupación se registre en sectores de la economía que demandan fuerza de trabajo poco calificada y mal remunerada. Estos segmentos empobrecidos de la clase trabajadora reúnen los requisitos para acceder a las prestaciones asistenciales. Del estudio realizado por el MIDES en 2011 sobre los hogares más pobres se desprende que "el $80 \%$ trabajaba, pero en el sector informal"'. En ese sentido, la calidad del trabajo (formalización y montos salariales) es lo que aparece como principal problema en un contexto de baja desocupación y crecimiento económico.

3. Disminución de la indigencia y reducción de la pobreza. En el primer caso, el resultado parece responder directamente al efecto de las transferencias de renta condicionada mientras que la reducción de la pobreza debe más a las mejoras salariales, a la reducción del desempleo y al combate a la informalidad. Este conjunto de medidas ha permitido mejorar las condiciones de vida de ciertos segmentos de la clase trabajadora. Logro de este período es la reducción del índice de Gini, aunque es necesario reconocer los límites de este indicador que, basado en la información que reúne la Encuesta Continua de Hogares del Instituto Nacional de Estadística, no recoge "la mayor parte de los ingresos del capital y por lo tanto sus resultados subestiman la concentración del ingreso y transmiten un mensaje confuso" (Notaro, 2012, p. 12). Si bien los efectos sobre la indigencia y la pobreza medidas por ingreso son visibles, los atributos duros de la pobreza medidos por necesidades básicas no han registrado modificaciones sustantivas. Se podría concluir, entonces, que los efectos se corresponden con una coyuntura económica favorable que, sumado a mecanismos regulatorios, alivió los déficits de ingresos en los hogares más pobres que incrementaron su capacidad de consumo. Sin embargo, queda en el debe el rebatimiento de la pobreza y la indigencia en dimensiones que, sin duda, requieren de políticas sostenidas en el tiempo y cuyos efectos no son inmediatos (educación, salud, vivienda, trabajo).

4. Consolidación de la racionalidad gerencial en la administración del Estado. Una racionalidad que apela a la tecnificación como forma de controlar

6. Disponible en: http://www.presidencia.gub.uy/comunicacion/comunicacionnoticias/programasprioritarios-olesker-mides-necesidades-poblacion-objetivo-jovenes-red-siete-zonas. Acceso en: 8 mayo 2015. 
los ingresos y egresos del Estado cuando de población "cautiva" se trata (asalariados y beneficiarios de prestaciones y servicios sociales). Sin embargo, estos cuidados con los dineros públicos se vuelven más laxos cuando se liberan zonas francas, se exhonera al capital o se avanza tímidamente en las imposiciones tributarias a las ganancias no salariales.

5. Inflación de las preocupaciones metodológico - instrumentales en el diseño de los programas. Parafraseando a Bourdieu (2000), una nueva "vulgata planetaria" se ha impuesto en el campo de las políticas sociales: sistemas de información, georreferenciamiento, proximidad o cercanías, territorialidad, trayectorias, transversalidad. Hipotéticamente se podría afirmar que esta inflación de la "técnica" se acompaña de un desplazamiento - o al menos de una minimización - de las preocupaciones políticas. En otras palabras, pareciera que el mensaje transmitido afirmara que si antes las cosas no iban bien era porque los principios de "derecha" orientaron la política pero ahora, que es la "izquierda" quien la formula, el problema se desplaza hacia aspectos metodológico - instrumentales y hacia la calidad del trabajo técnico-profesional.

6. Las prestaciones asistenciales han crecido en calidad, cantidad y cobertura. Sin embargo, la ayuda social tiende a permearse y/o combinarse con políticas punitivas dirigidas a estas poblaciones. Se observa una riesgosa reedición de la asociación entre políticas asistenciales y punitivas en los territorios de la pobreza o, en otros términos, la reasociación organizacional de la mano derecha y la izquierda del Estado (Wacquant, 2011). Menciono algunos ejemplos a título ilustrativo:

i) La creación de ámbitos de coordinación institucional con participación de los Ministerios con competencias en materia de seguridad pública y asistencia y protección social. En 2010, el Ministerio del Interior se integra al Gabinete Social, al Consejo Nacional de Políticas Sociales y a las Mesas Interinstitucionales de Políticas Sociales. Estas instancias tienen como cometido la definición de acciones estatales "en materia de políticas públicas de protección social". ${ }^{7}$ Además se crea el Gabinete de Seguridad Pública que en 2012, con el MIDES integrado,

\footnotetext{
7. Disponible en: http://presidencia.gub.uy/comunicacion/comunicacionnoticias/ministerio-interior-avances-ultimo-quinquenio-incorporaciones-tecnologia-personal. Acceso en: 11 mayo 2015.
} 
formula la Estrategia por la vida y la convivencia con el objetivo de articular las políticas de seguridad con las sociales.

ii) El Plan 7 Zonas es producto de la acción conjunta de los Ministerios del Interior y Desarrollo Social en siete zonas seleccionadas por sus "altos niveles de violencia e inseguridad y (...) con indicadores sociales de alta exclusión persistente" (Ministerio del Interior, 2014). Sus propósitos son mejorar la focalización en zonas pobres, desarrollar un plan de mejoras urbanas para la "convivencia" e implementar un nuevo diseño del sistema de patrullaje y seguridad en dichas zonas (Ministerio del Interior, 2014).

iii) La Ley de Faltas en su implementación demanda la coordinación de ambas carteras ministeriales. Esta normativa regula y sanciona comportamientos sociales en ámbitos diversos: espacios públicos, tránsito, convivencia etc. Parte de la regulación se dirige a un conjunto de comportamientos atribuidos a los pobres (ocupación de espacios públicos, mendicidad y otras estrategias de generación de ingresos en el espacio urbano). El protocolo de intervención policial establece que los funcionarios policiales deben intimar a la persona a abandonar la ocupación indebida de espacios públicos, trasladarla a una dependencia policial, registrar sus datos en el Sistema de Gestión de la Seguridad Pública en la tipificación de la falta referida, comunicar al juez y derivar las situaciones al MIDES a fin de procurar su resolución ${ }^{8}$.

iv) De más reciente aparición y de corta vida, pues al parecer ha sido desechado, es el "Protocolo de seguimiento y actuación para garantizar el derecho a la educación”, aprobado en febrero de 2015. El protocolo establece que, una vez "agotados" los esfuerzos del centro educativo para lograr la inserción o reinserción del niño o adolescente al sistema educativo, se solicitará la intervención policial a fin

8. “'No sé por qué de éstos que reincidieron no hay ningún procesado', se limitó a informar Fernando Gil a El País, quien redactó el informe del Ministerio del Interior. Consultado el vocero de la Suprema Corte de Justicia, Raúl Oxandabarat, explicó que la persona en situación de calle no puede ser procesada si el Estado no le brindó una solución a su situación (...) El País se comunicó con el Ministerio de Desarrollo Social, con el fin de saber si, efectivamente, se le brindó soluciones alternativas a las personas que son reincidentes en la ocupación de espacios públicos (...) 'Estas personas son valoradas médicamente, y en una primera instancia, si lo desean, pero sólo si lo desean, se les ofrece pernoctar en los distintos centros que tenemos, ya sea nocturnos o de 24 horas, además de darles comida. Pero el MIDES no los detiene, no están presos allí. Solo si ellos lo desean pueden optar por dormir bajo techo, en un espacio que les brinda el Ministerio', indicó la fuente a El Pais". Disponible en: http://www.elpais.com.uy/informacion/indigentes-dia-son-detenidos-policia.html. Acceso en: 26 ene. 2015. 
de "hablar con los padres o adultos responsables del niño afectado e intimarlos a regularizar la asistencia al centro educativo".

\section{Hacia una interpretación general sobre la orientación de la política social en el período}

Una interrogante previa al intento de interpretar la orientación de la política social en el ciclo de recuperación posneoliberal en América Latina es ineludible: ¿cuál es el sentido atribuido a la expresión "ciclo de recuperación posneoliberal"? Entiendo por "posneoliberalismo" una nueva metamorfosis del proyecto neoliberal que lleva implícita una reingeniería del Estado necesaria, entre otras cosas, para administrar los efectos sociales regresivos de la libre competencia. La desregulación de la competencia en todas las esferas de la vida social como principal estrategia del proyecto neoliberal continúa vigente. En ese sentido, el posneoliberalismo no logra consolidarse como la negación y la superación de esta estrategia.

Los últimos diez años (o los primeros diez años) de la izquierda en el gobierno han estado marcados por fuertes y esperables contradicciones. La propuesta analítica de Wacquant (2011) - construida sobre el arsenal heurístico heredado de Bourdieu - me parece apropiada para analizar estas contradicciones como expresión de las luchas en los campos burocrático y político. Pensar la correlación de fuerzas como luchas entre la mano derecha y la izquierda del Estado, así como entre la alta y la baja nobleza estatal es el camino para comprender estas contradicciones, evaluar la dinámica de estas luchas y sus — eventuales y provisorios — ganadores y perdedores.

En ese sentido, se comparten las apreciaciones de Narbondo (2012, pp. 330 y ss.) quien advierte dos grandes tendencias en las reformas socioeconómicas impulsadas en el período. La primera tendencia se caracteriza por representar la "aceptación pragmática del capitalismo neoliberal como asignador más racional de las inversiones y conductor de la economía” y se limita a

9. Administración Nacional de Educación Pública, Consejo Directivo Central, Circular n. 31/2014. 
la "reproducción de un sistema de Bienestar focalizado y residual". La segunda tendencia mantiene orientaciones claramente antineoliberales e insiste en la necesidad de un sistema de bienestar universalista, con mayor participación del Estado para estimular un desarrollo con mayor valor agregado nacional. Como resultado históricamente provisorio de estas luchas asistimos hoy a un limitado retorno del Estado que es expresión de una reingenieria del modelo en un país que, como decíamos al comienzo, supo aproximarse a la construcción de una "sociedad amortiguadora".

La experiencia uruguaya indica, a mi entender, que los dos gobiernos de izquierda han promovido un "leve" retorno del Estado como parte de la reingeniería del Estado. El Estado contemporáneo no reniega de su papel garante en la producción y distribución de determinados bienes y servicios públicos sociales. Pero, conserva trazos neoliberales en la medida en que "no busca expandir y ampliar la desmercantilización de la sociedad y de la economía, sino por el contrario, busca limitarla a lo estrictamente necesario para la maximización de los ámbitos de producción de bienes y servicios mercantilizados" (Narbondo, 2011).

De este modo, y coincidiendo con Antía (et al., 2013), los sectores sociales mejor posicionados recurren al mercado y a las unidades estatales que mejor funcionan para administrar sus riesgos sociales mientras que los segmentos más empobrecidos permanecen en la esfera pública: "se abandona explícitamente la pretensión política de cubrir las necesidades de los diversos grupos sociales por los mismos principios e instituciones, consolidando con recursos públicos un esquema dual de bienestar" (p. 190).

Como se ha dicho, en este período el Estado rediseña sus estrategias y modalidades de regulación e intervención. El debate, entonces, gira en torno a la orientación de esta reingeniería: en cuanto se aparta o reproduce los contenidos del proyecto neoliberal $\mathrm{o}$, en otras palabras, qué tanto han mejorado las condiciones de reproducción de la clase trabajadora. Parte del pensamiento político de izquierda vió en la llegada del Frente Amplio al gobierno la posibilidad histórica de avanzar hacia la construcción de una sociedad menos desigual recuperando parte del poder domesticador del Estado sobre el capital. La brecha entre estas expectativas, por un lado, y la moderación y contradicciones 
del proceso real de transformaciones, por el otro, se reproduce en el seno del Estado contorneando los espacios donde se expresa la puja por la orientación de la acción estatal.

La idea del "retorno" del Estado en la era progresista suele apoyarse, entre otros elementos, en la significación sociopolítica de la creación del MIDES. El énfasis que la izquierda en el gobierno le ha atribuido a las políticas de asistencia ha sido señalado como un reconocimiento de las debilidades del libre mercado para dar cuenta de las situaciones de pobreza e indigencia (Midaglia, 2012). La reformulación del régimen de Asignaciones Familiares (AFAM) mejorando la prestación, desanclándola del mundo del trabajo y extendiendo su cobertura es presentada como el principal indicador de lo antedicho. Sin embargo, el modelo bipolar (contributivo - no contributivo) de las AFAM es anterior a los gobiernos de izquierda los que, sin duda, mejoraron la prestación y extendieron su cobertura. También hay que reconocer que la renovada preocupación por la asistencia recorre el continente con independencia del signo político de los gobiernos latinoamericanos. En síntesis, el Estado nunca estuvo ausente: como dice Castel (1997), el "retiro" del Estado no es más que una metáfora para ilustrar las alteraciones en sus objetos y modalidades de intervención.

Respecto a los principales atributos de la política social neoliberal, se observan rastros de continuidad en el período estudiado, con variaciones discursivas y formas más o menos novedosas de nominar prácticas no tan nuevas. Sobre la asociación público-privada para la prestación de servicios sociales no hubo alteraciones sustantivas; se continuó con esta estrategia asociativa y, quizás lo más notorio, ha sido el mayor esfuerzo del Estado, en particular en algunos programas, por orientar políticamente la administración de los servicios cogestionados.

El énfasis puesto a la "dimensión territorial" de la política social ha estado persistentemente presente en la producción discursiva y en el diseño de estategias de intervención ancladas en los territorios de la pobreza y con metodologías de proximidad o cercanías.

La focalización dejó de ser un "demonio" para convertirse en un "instrumento para priorizar la asignación de recursos con un carácter subsidiario de una política pública más universalista" con el objetivo de "permitir el tránsito 
como si fuera una carretera desde las acciones focalizadas (estrategia de inclusión) hacia los servicios universales (estrategia de integración)" (MIDES, 2015, p. 164). Pese a la nueva retórica acerca de la focalización, se observa que, hasta el momento, esta orientación de la política ha contribuido a reforzar la dualización del sistema de protección. En la medida en que las intervenciones operan como atenciones paralelas dirigidas a los más pobres (Sposati, 2011, p. 111), la pretendida "estrategia de integración" está aún en el debe.

"El consenso neoliberal echó raíces", nos recuerda Sader (2008, p. 78); el neoliberalismo triunfó culturalmente, nos advierte Grassi (2004) y Wacquant (2011, p s/d) agrega que estamos frente a un proyecto "al que adhieren indistintamente los políticos de derecha y de izquierda". Las señales políticas recientes parecen confirmar, no sin contradicciones, este provisorio triunfo y es éste, quizás, el principal debe de la izquierda en el gobierno que no ha sabido, podido o querido conducir un discurso contrahegemónico limitándose a reproducir el existente. En ese sentido, las señales políticas de la izquierda en el gobierno han sido fuertemente contradictorias: sobre una retórica anclada en la idea de derechos se hiperfocalizan las prestaciones sociales y se demanda "apretar en las contrapartidas exigibles" por "el respeto que merecen los recursos públicos que suda la gente" 10 . Abundan ejemplos de este tipo pero es suficiente para ilustrar lo que quiero transmitir.

Por otro lado, hay avances notorios en la politización de "terrenos hasta entonces considerados apolíticos (feminismo, políticas gay y lesbiana, ecología, problemas de minorías étnicas y otras)" (Žižek, 2001) y menos significativos en el terreno de democratizar desmercantilizando, es decir, retirando de la esfera del mercado para pasar a la esfera del derecho (Sader, 2008).

Aquí hay otro desafío central para un gobierno de izquierda y para la acción colectiva. Se podría formular como un principio estratégico que aquello que no se logra obtener del capital mediante la política salarial habría que obtenerlo a través de más y mejores prestaciones sociales. Una dificultad, en ese sentido, es la relativa invisibilidad que tiene la política social en general, pero

10. Disponible en: http://www.mides.gub.uy/innovaportal/v/20739/3/innova.front/del_lado_de_la_convivencia. Acceso en: 10 jul. 2013. 
fundamentalmente la política asistencial, como espacio de lucha para el movimiento obrero. El carácter estructuralmente residual de la asistencia, pensada siempre en paralelo a la seguridad social, contribuye a esta externalidad del movimiento obrero respecto a su devenir. A su vez, la maximización de la mercantilización de bienes y servicios para la administración de los riesgos sociales, la huída hacia el mercado de los sectores medios y altos (en áreas estratégicas como la educación y la salud), la hiperfocalización en la pobreza y la disociación de las prestaciones del mundo del trabajo configuran un escenario complejo para mejorar la oferta de prestaciones a través de la acción colectiva.

Más sombrío se vuelve el panorama si observamos las señales políticas del último escenario electoral. Las propuestas de la oposición política al gobierno corren, decididamente y como es de esperar, por la derecha. Las principales críticas formuladas desde la oposición se concentran en la necesidad de reducir el tamaño del Estado, recuperar el equilibrio fiscal ajustando el gasto público, repensar la matriz de protección social promoviendo políticas de workfare, mantener la "imparcialidad" del Estado en las negociaciones colectivas etc.

Si bien estas reflexiones tienen un carácter inacabado, entiendo que los esfuerzos por avanzar hacia una comprensión acabada y sin concesiones de este escenario, que no deja de ser coyuntural, es condición indispensable para su superación.

Recebido em 23/6/2016 - Aprovado em 13/2/2017

\section{Referencias bibliográficas}

ADMINISTRACIÓN NACIONAL DE EDUCACIÓN PÚBLICA. Protocolo de seguimiento y actuación para garantizar el derecho a la educación. Circular n. 31/2014, 2015. ANTÍA, F.; CASTILLO, M.; FUENTES, G.; MIDAGLIA, C. “La renovación del sistema de protección uruguayo: el desafío de superar la dualización.” Revista Uruguaya de Ciencia Política. Montevideo, n. 22, Instituto de Ciencia Política/UdelaR, 2013. 
BENTURA, J. P. “Los programas de transferencia de renta condicionadas como gestión neoliberal de la cuestión social”. Serviço Social e Sociedade. São Paulo: Cortez, n. 117, p. 94-121, jan./mar. 2014.

BENTURA, P.; MARIATTI, A. Gasto público social y presupuesto de las Asignaciones Familiares del Plan de Equidad. Informe preliminar de investigación (mimeo.), 2014.

BOURDIEU, P. La nueva vulgata planetaria. Disponible en: http://www.pedagogica. edu.co/storage/rce/articulos/42_04ens.pdf. Acceso en: 25 feb. 2017, 2000.

. Génesis y estructura del campo burocrático. Disponible en: http://190.110.216.14/ librosdig/todos/Bourdieu, \%20P\%20-\%20Genesis\%20y\%20estructura\%20del\%20 campo\%20burocratico.pdf. Acceso en: 25 feb. 2017, 1993.

BUSTELO, P. Desarrollo económico: del Consenso al Post-Consenso de Washington y más allá. Disponible en: http://pendientedemigracion.ucm.es/info/eid/pb/BusteloPCW03. htm. Acceso en: 25 feb. 2017, 2003.

CASTEL, R. De la peligrosidad al riesgo. En: Materiales de Sociología Crítica. Madrid: Ediciones de La Piqueta, p. 219-243, 1986.

Las metamorfosis de la cuestión social. Una crónica del salariado. Buenos Aires: Paidós, 1997.

. Propiedad privada, propiedad social, propiedad de sí mismo. Conversaciones sobre la construcción del individuo moderno. Rosario (Argentina): Homo Sapiens, 2003.

COMITÉ DE COORDINACIÓN ESTRATÉGICA. Estrategia Nacional para la Infancia y la Adolescencia (2006-2010), nov. 2005. Acceso en: 5 mayo 2015.

GRASSI, E. Política y cultura en la sociedad neoliberal: la otra década infame II. Buenos Aires: Espacio Editorial, 2004.

INSTITUTO CUESTA DUARTE. Informe de Coyuntura Primer trimestre de 2015. Disponible en: http://cuestaduarte.org.uy/investigacion/economia/informes-decoyuntura/item/64-informe-de-coyuntura-primer-trimestre-de-2015. Acceso en: 25 feb. $2017,2015$.

MERKLEN, D. Conversaciones sobre lo público. Entrevista realizada por la Dra. Claudia Danani. Revista Debate Público. Reflexión de Trabajo Social. Año 3, n. 6. Argentina. Disponible en: http://trabajosocial.sociales.uba.ar/web_revista_6/PDF/03_Merklen.pdf. Acceso en: 25 feb. 2017, 2013. 
MIDAGLIA, C. Un balance crítico de los programas sociales en América Latina. Entre el liberalismo y el retorno del Estado. Revista Nueva Sociedad, n. 239, 2012.

. Alternativas de protección a la infancia carenciada. Colección Becas de Investigación CLACSO, 2000.

. El rendimiento de los "by-pass" como instrumento de reforma social: el caso PRIS. Revista Uruguaya de Ciencia Política, n. 10. Montevideo: Instituto de Ciencia Política, 1998.

MIDAGLIA, C. et al. El significado político de los Ministerios Sociales en Argentina, Chile y Uruguay. Revista Chilena de Administración Pública, n. 15-16, p. 123-154, 2010.

MINISTERIO DE DESARROLLO SOCIAL. Plan de Equidad. Disponible en: http:// www.mides.gub.uy/innovaportal/file/913/1/plan_equidad_def.pdf. Acceso en: 20 ago. 2009, 2007.

MINISTERIO DE DESARROLLO SOCIAL. Estrategia Nacional de Fortalecimiento de las Capacidades Familiares. Programa Cercanías, 2013.

MINISTERIO DE DESARROLLO SOCIAL. Informe de Transición. Disponible en: http://www.mides.gub.uy/innovaportal/file/40855/1/mides_informe-detransicion-2011-2015.pdf. Acceso en: 28 mar. 2015, 2015.

MINISTERIO DEL INTERIOR. Evaluación de indicadores de seguridad pública en las zonas de intervención del plan 7 zonas. Disponible en: https://www.minterior.gub. uy/images/7zonas.pdf. Acceso en: 12 jun. 2014.

MOREIRA, C. "La Reforma del Estado en Uruguay: cuestionando el gradualismo y la heterodoxia." Calamé y Talmant, Con el Estado en el corazón. El andamiaje de la gobernancia. Montevideo: Trilce, 2001.

NARBONDO, P. “¿Estado desarrollista de bienestar o construcción de la izquierda del Estado neoliberal? Los gobiernos del Frente Amplio de Uruguay.” En: THWAITES REY, M. El Estado en América Latina: continuidades y rupturas. Buenos Aires: Consejo Latinoamericano de Ciencias Sociales, Colección Clacso, 2012.

. "Contratos gerencialistas, Estado neoliberal y autonomía enraizada." Revista Uruguaya de Ciencia Politica, v. 20, n. 1. Montevideo. Disponible en: http://www. scielo.edu.uy/scielo.php?pid=S1688-499X2011000100005\&script=sci_arttext\&tlng=en. Acceso en: 25 feb. 2017, 2011. 
NOTARO, J. La distribución social del ingreso en Uruguay. Diagnóstico y propuestas. Cuadernos del CLAEH, n. 100. Montevideo, 2012.

ORLANSKY, D. El concepto de Desarrollo y las Reformas Estatales: Visiones de los Noventa. Disponible en: www.iigg.fsoc.uba.arsepurePublicacionesOrlansky.pdf. Consulta: 22/4/09. Acceso en: 25 feb. 2017, 2005.

ORTEGA, E. El servicio social y los procesos de medicalización de la sociedad uruguaya en el período neobatllista. Rio de Janeiro/Montevideo: Universidad Federal de Río de Janeiro/Universidad de la República. Tesis de Maestría, 2003.

SADER, E. Refundar el Estado. Posneoliberalismo en América Latina. Disponible en: http://bibliotecavirtual.clacso.org.ar/ar/libros/coedicion/sader/sader.pdf. Acceso en: 25 feb. 2017, 2008.

SERNA, M. El malestar ciudadano con el reformismo uruguayo de los noventa. Revista de Ciencias Sociales, año XVIII, n. 22. Montevideo: Departamento de Sociología, Facultad de Ciencias Sociales, Universidad de la República, 2005.

SPOSATI, A. Tendências latino-americanas da política social pública no século 21. Rev. Katálysis, vol. 14, n. 1. Florianópolis, 2011.

VECINDAY, L. La reconfiguración del campo asistencial. El caso del Plan de Equidad de Uruguay. Serviço Social \& Sociedade, São Paulo, n. 116, out./dez. 2013.

WACQUANT, L. Forjando el Estado neoliberal. Workfare, Prisonfare e Inseguridad Social. Revista Prohistoria, n. 16, Rosario (Argentina), jul./dic. 2011.

ŽIŽEK, S. El espinoso sujeto. El centro ausente de la ontología política. Buenos Aires: Paidós, 2001. 